PO038

\title{
PILOT STUDY ON COLOR MATCHING ACCURACY USING DIFFERENT PRIMARIES
}

\author{
Jiaye Li et al. \\ DOI 10.25039/x46.2019.PO038 \\ from \\ CIE x046:2019 \\ Proceedings \\ of the \\ 29th CIE SESSION \\ Washington D.C., USA, June 14 - 22, 2019 \\ (DOI 10.25039/x46.2019)
}

The paper has been presented at the 29th CIE Session, Washington D.C., USA, June 14-22, 2019. It has not been peer-reviewed by CIE.

(C) CIE 2019

All rights reserved. Unless otherwise specified, no part of this publication may be reproduced or utilized in any form or by any means, electronic or mechanical, including photocopying and microfilm, without permission in writing from CIE Central Bureau at the address below. Any mention of organizations or products does not imply endorsement by the CIE.

This paper is made available open access for individual use. However, in all other cases all rights are reserved unless explicit permission is sought from and given by the $\mathrm{CIE}$.

CIE Central Bureau

Babenbergerstrasse 9

A-1010 Vienna

Austria

Tel.: +4317143187

e-mail: ciecb@cie.co.at

www.cie.co.at 


\title{
PILOT STUDY ON COLOR MATCHING ACCURACY USING DIFFERENT PRIMARIES
}

\author{
Li, J. ${ }^{1}$, Hanselaer, P. ${ }^{2}$, Smet, K.A.G. ${ }^{1 *}$ \\ ${ }^{1} \mathrm{KU}$ Leuven, ESAT/Light\&Lighting Laboratory, Ghent, BELGIUM \\ *kevin.smet@kuleuven.be
}

DOI 10.25039/x46.2019.PO038

\begin{abstract}
Over time, much work has been carried out to ascertain the accuracy of the CIE standard colormatching functions (CMF) and no definitive answer has been given. Recent work indicates an undeniable discrepancy between visual and computed metamers calculated using the existing CIE (the International Commission on Illumination) standard observer CMFs, especially when narrowband sources are involved. A series of pilot matching experiments using narrow band primaries with different peak wavelengths has been done to identify which regions in wavelength space are most sensitive to generate matching inaccuracies for a given CMF set and to determine the most accurate set. The derivation of an improved colorimetric system would have important consequences not only for the prediction of color (mis)matches, but also for all colorimetric calculations based on CMFs.
\end{abstract}

Keywords: Color Matching, Color Matching Functions, Matching primaries, Observer Variability

\section{Introduction}

Good color description starts with accurate color matching functions (CMFs) or cone fundamentals (CF). However, it has become increasingly apparent that there are substantial discrepancies between visual matches and the matches calculated using the 1931 (and 1964) CMFs defined by the International Commission on Illumination (CIE), especially for narrowband sources such as solid-state and laser based sources [Csuti 2008, 2010].

This paper reports on the results of a series of pilot matching experiments using narrowband primaries with different peak wavelengths, generated by different channels of a spectrally tunable LED light source. The aim is to identify which regions in wavelength space are most sensitive to induce matching inaccuracies for a given CMF set and to determine the most accurate set of CMFs.

\section{Methods}

\subsection{Apparatus}

Matching was done in a specially designed viewing box, allowing the matching field (where the spectrally tunable stimuli were presented) to be viewed in a reflective / object appearance mode and in a dark or luminous surround. An overall configuration of the apparatus is shown in Figure 1. 


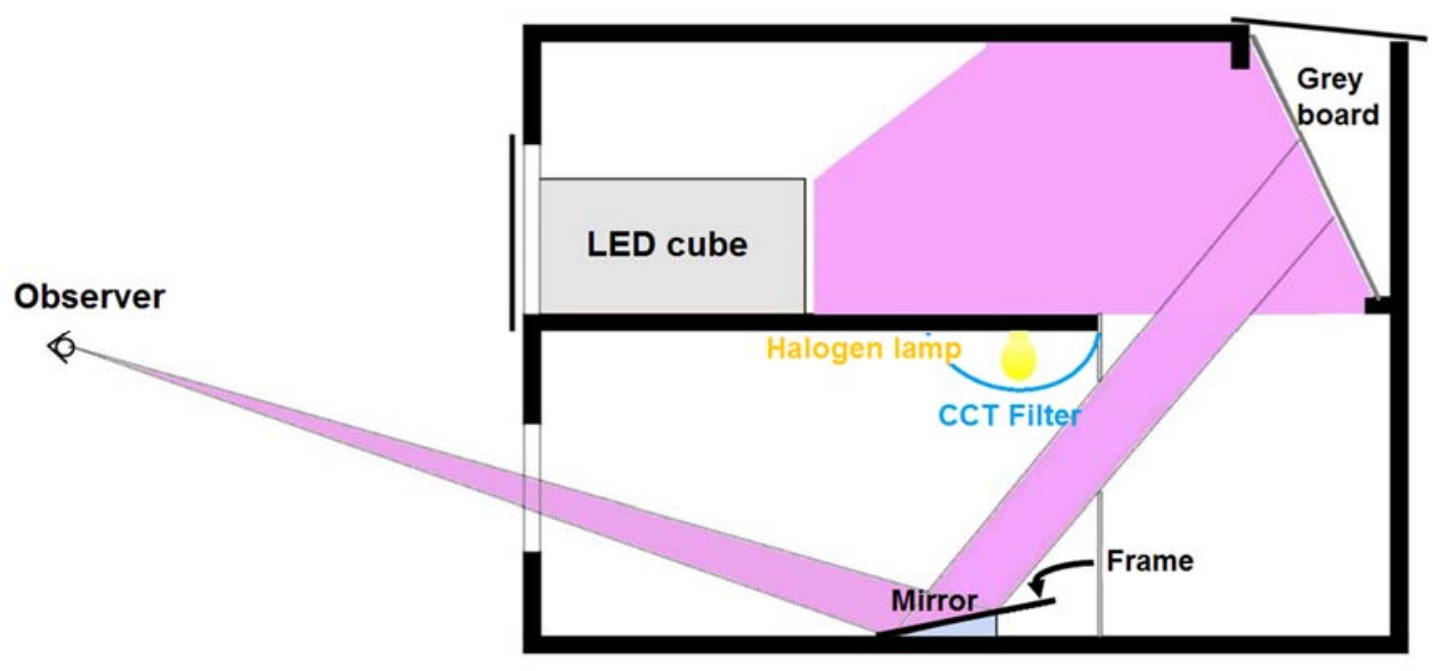

Figure 1 - Apparatus: Overall configuration of viewing booth

In the box, a tilted mirror ( $3^{\circ}$ field of view) was used to provide the matching field. By carefully positioning the observer, the mirror showed only the reflected image of a neutral grey plate illuminated by a calibrated ThousLite LED cube. The LED cube with 15 10-bit channels provides different matching primaries. The grey plate and the LEDcube were positioned in a separate compartment of the viewing box to avoid contributing to the background and surround of the matching field. The upper chamber was covered by spectrally flat, non-UV-enhanced, white material to provide optimal mixing. By changing the illumination of the LEDcube the apparent color of the matching field changed as well. Pilot experiments have shown that this mirror setup can provide a stimulus which is indistinguishable from an illuminated opaque, reflective card covered by a glass plate, of the same luminance.

For the experiments reported in this paper, a dark surround was generated by covering it with a minimally reflecting black cloth.

In a first series of experiments, observers were asked to make achromatic (neutral grey) matches based on memory and the immediate background was also set to black using the same cloth.

In a second series of experiments, observers were asked to match the color appearance of the immediate frame of the mirror. For these experiments, the mirror frame ( $5^{\circ}$ field of view) was composed of a neutral (spectrally flat) grey material and was illuminated by a $50 \mathrm{~W}$ halogen source. The halogen source was filtered by a flexible blue filter (CCT conversion filter: LEE filter 201) to obtain a uniform neutral reference illumination with a chromaticity slightly above the blackbody locus at around $5000 \mathrm{~K}$. The luminance of the reference illumination was set at 40 $\mathrm{cd} / \mathrm{m}^{2}$, a representative lighting level of building interiors [Houser 2005]. Because of the tilt of the mirror, observers, seated in a fixed position, did not see the halogen, but only the mirror image of the reflection plate. The set-up is shown in Figure 1.

The spectral radiance of each match made by an observer was measured with an Ocean-optics tele-radio spectrometer after suitable calibration.

\subsection{Matching primaries and starting points}

Matching primaries were generated by the LED cube. Six channels were chosen as primaries: two long wavelength (red), two medium wavelength (green) and two short wavelength (blue). The details of each primary are shown in Table 1: peak wavelength, full-width-half-maximum and maximum radiance when driven at maximum current as measured from the observer's position. The mutual independent primaries were chosen to provide a large gamut size at 40 $\mathrm{cd} / \mathrm{m}^{2}$ (see Fig. 2) and to have a substantial gap in wavelength space between their peaks. The selected primaries were combined in 6 different primary sets, each composed of one long, one medium and one short wavelength primary. 
Table 1 - Overview of chosen primaries

\begin{tabular}{|c|c|c|c|c|}
\hline \multicolumn{2}{|c|}{ Primary } & Peak wavelength (nm) & $\begin{array}{c}\text { Full-width-half-maxima } \\
(\mathrm{nm})\end{array}$ & $\begin{array}{c}\text { Maximum } \\
\text { radiance } \\
\left(\mathrm{W} / \mathrm{m}^{2} . \mathrm{sr}\right)\end{array}$ \\
\hline \multirow{2}{*}{$\begin{array}{c}\text { Long } \\
\text { wavelength }\end{array}$} & Red 1 & $635,7 \mathrm{~nm}$ & 19 & 31,42 \\
\hline \multirow{2}{*}{$\begin{array}{c}\text { Medium } \\
\text { wavelength }\end{array}$} & Red 2 & $597,7 \mathrm{~nm}$ & 16 & 24,05 \\
\hline \multirow{2}{*}{$\begin{array}{c}\text { Short } \\
\text { wavelength }\end{array}$} & Green 2 & $521,3 \mathrm{~nm}$ & 34 & 88,80 \\
\cline { 2 - 5 } & Blue 1 & $502,4 \mathrm{~nm}$ & 29 & 75,17 \\
\hline
\end{tabular}

To minimize the starting point bias when matching, 5 different starting chromaticities were chosen based on the $5 \mathrm{Y}, 5 \mathrm{G}, 5 \mathrm{~B}, 5 \mathrm{P}$ and $5 \mathrm{R}$ Munsell hue (black dots) and having the same distance to the D65 reference white point. The starting points are shown in Figure 2.

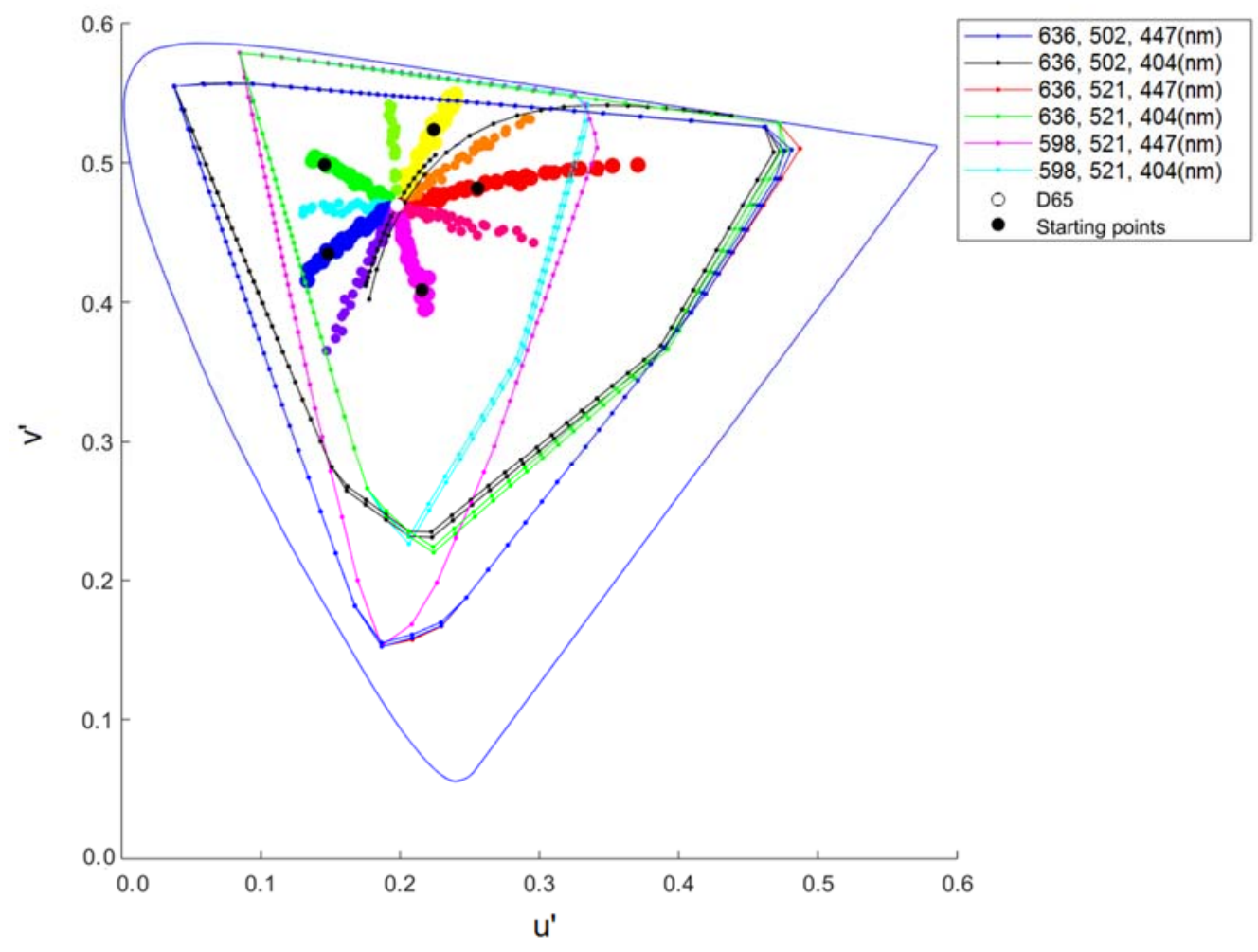

Figure 2 - Color gamuts of the 6 selected sets of primaries sets at $40 \mathrm{~cd} / \mathrm{m}^{2}$ in the CIE $1976 u^{\prime} v^{\prime}$ chromaticity space diagram. Starting points (black dots) distributed evenly on the Munsell hue lines (indicated by the red, yellow, green, blue and violet solid circles) and having the same distance to the D65 reference white point (white dot) are also shown.

\subsection{Experimental procedure}

The matching experiments were subdivided into two sessions ( 2 matching paradigms): in the first experimental session, observers were asked to make achromatic matches from memory (memory achromatic matching), while in the second session, observers were asked to make a simultaneous match with the grey mirror frame, illuminated by the filtered halogen, as reference color. Note that the grey mirror frame has a smooth broad-band reflection spectrum and had the same luminance as the matching field $\left(40 \mathrm{~cd} / \mathrm{m}^{2}\right)$.

Before each matching session started, the experimenter gave each observer a brief instruction on the goal of the study and on the experimental procedure and the observer had the occasion to learn how to change the matching stimuli by using the keyboard. Three matching test trials were provided for each observer. The results of these test trials were not included in the analysis. 
During the first experiment, the observers were asked to adjust the apparent color of the mirror area to neutral grey by navigating in the CIE 1976 u'v' $^{\prime}$ space using a keyboard. After finishing a match, they were asked to rate their result on a 0 (not satisfied at all) to 10 (very satisfied) scale.

For each primary set, observers were asked to make 5 matches, each starting from a different initial chromaticity to minimize starting bias. Primary sets and starting points were all fully randomized for each observer to avoid order bias.

The luminance of matching field, the matching primary-sets, starting stimuli, and field of view of matching field (mirror and mirror frame) were kept the same for the two sessions.

\subsection{Observers}

8 observers ( 5 females, 3 males, average age: $24 \pm 4$ years) were invited to this experiment, all with normal colour vision, as tested by the Ishihara 24-plate test. The same observers participated in both experiment sessions.

\section{Results and discussion}

During the experiments, a total of 480 estimations were made: 8 observers $\times 2$ experiment sessions (memory color matching and simultaneous matching) $\times 6$ primary-sets $\times 5$ starting points. Results were analysed per experiment session: memory matching (MM) and reference matching (RM).

For each primary-set, eighty matches were made in each experiment session. The chromaticity of the matches was calculated using the CIE $19312^{\circ}$ [CIE15-2004 2004], CIE $196410^{\circ}$ [CIE152004 2004], and $\mathrm{CIE} 20062^{\circ}$ and $10^{\circ} \mathrm{CMFs}$ [CIE 2006]. Figure 3 and Figure 5 show the mean chromaticity of these matches (made by each primary-set) and the standard error (SE) ellipses around the matches on the CIE 1976 U'v' diagram for the memory color matching and reference matching conditions, respectively. The use of different CMFs is indicated by different plot colors.

For the memory matching condition (MM), as can be seen from Fig. 3, different primary sets result in different $u^{\prime} v$ ' distributions of the mean match. Although the SE ellipses for each primary-set are approximately equal sized and have approximately the same orientation, their locations are substantially different, except for the primary-set with peak wavelengths at 636 $\mathrm{nm}, 521 \mathrm{~nm}$, and $447 \mathrm{~nm}$. The close grouping of the matches for this primary set indicates that these primaries are less sensitive to generate metameric differences.

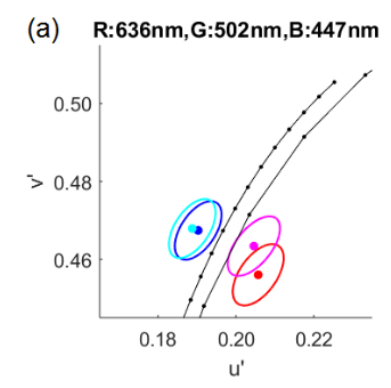

(d) R:636nm,G:502nm,B:404nm

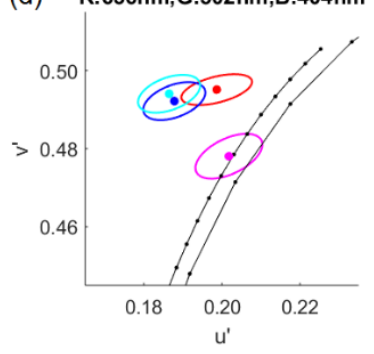

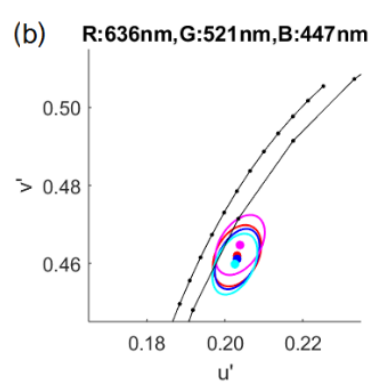

(e) R:636nm,G:521 nm,B:404nm

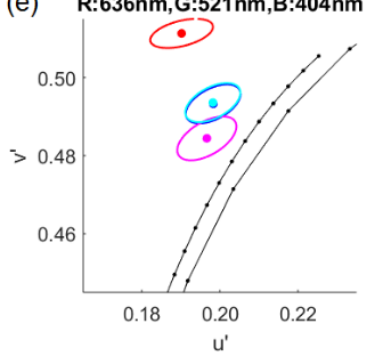

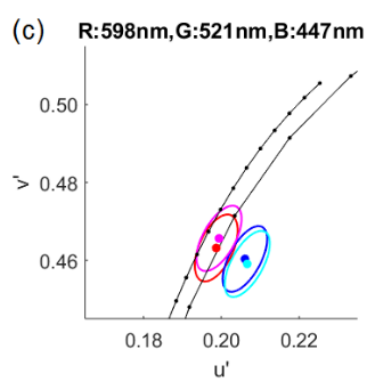

(f) R:598nm, G:521 nm,B:404nm

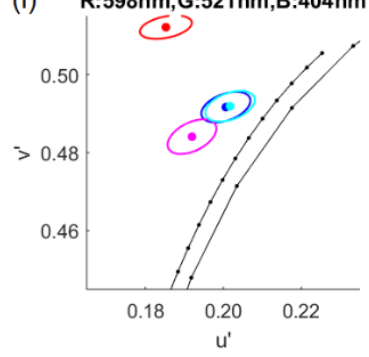

Figure 3-MM condition: Computed mean chromaticity coordinates of memory matches (dots) and their SE ellipses for different primary sets (subplots a-f) and CMF sets (Red: CIE $19312^{\circ}$ CMFs, Blue: CIE 1964 10 CMFs, Magenta: CIE $20062^{\circ} \mathrm{CMFs}$, Cyan: CIE $200610^{\circ} \mathrm{CMFs}$ ). 
Depending on which primary is replaced in this 'stable' set, the computed matches tend to shift in different directions in the $u^{\prime} v$ ' diagram. For the medium wavelength primary: replacing Green $1(521 \mathrm{~nm})$ with Green $2(502 \mathrm{~nm})$ caused a noticeable split along the $u^{\prime}$-direction between the chromaticity computed by the $2^{\circ} \mathrm{CMFs}$ and those computed by $10^{\circ} \mathrm{CMFs}$. The $2^{\circ}$ matches move to higher $u^{\prime}$ coordinates, while the $10^{\circ}$ matches move to lower $u^{\prime}$ coordinates. For the long wavelength primary: replacing Red $1(635 \mathrm{~nm})$ with Red $2(598 \mathrm{~nm})$ doesn't have a substantial influence on the $10^{\circ}$ matches, but results in a shift to lower $u$ ' coordinates for the $2^{\circ}$ matches. Furthermore, for the short wavelength primary: switching Blue $1(447 \mathrm{~nm})$ with Blue $2(404 \mathrm{~nm})$, causes a shift towards higher $v$ ' coordinates for all CMFs sets except the CIE 2006 $2^{\circ} \mathrm{CMFs}$, which remain quite stable.

Differences between primary sets for each CMF set are illustrated in Figure 4. It can be seen that the matches made with each primary set are differently distributed in the $u^{\prime} v^{\prime}$ diagram depending on the CMF set used to calculate them. Of the four CMF sets, the CIE $19312^{\circ} \mathrm{CMFs}$ and the CIE $20062^{\circ}$ CMFs show respectively the largest and the least discrepancy with the others. The two $10^{\circ}$ CMFs show very similar predicted matches, both in the position of the mean, as well as the size, shape and orientation of the SE ellipses. However, the CIE 1931 and CIEOP06 $2^{\circ}$ CMFs result in very different matches for many primary sets, especially for the primary sets containing the $404 \mathrm{~nm}$ primary (the upper group of ellipses in the first graph of Figure 4). This agrees with the underestimation of the sensitivities of the CIE 1931 CMFs at lower wavelengths reported in literature [Csuti 2008, 2010, Ezquerro 2008].
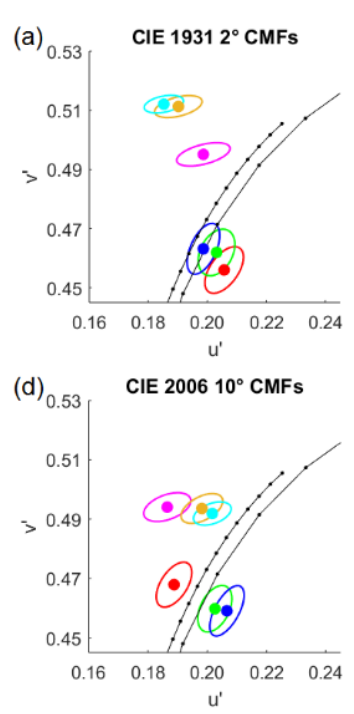
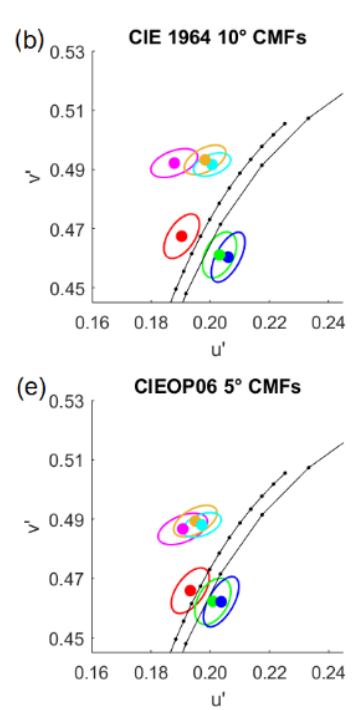
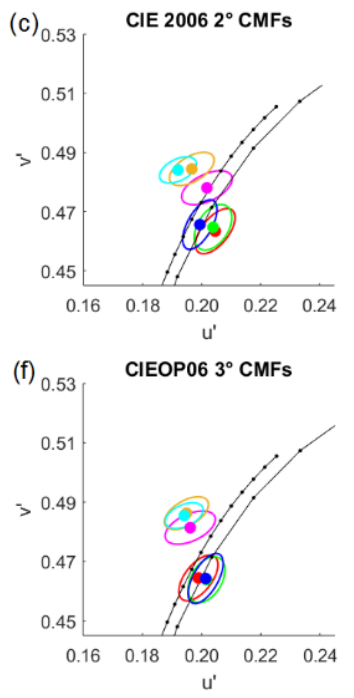

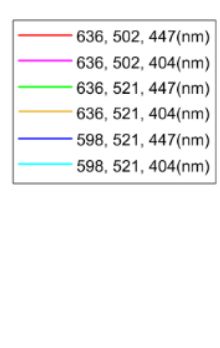

(1)

Figure 4-MM condition: Mean chromaticity coordinates of memory matches (dots) and their SE ellipses computed by different CMF (subplots a-f), and primary-sets (the six colors indicate the six different primary-sets).

The match ellipses calculated with the CIE $2^{\circ}$ CMFs are more closely clustered than those calculated with the $10^{\circ} \mathrm{CMFs}$. One reason maybe that the field size factor was taken into account in the CIEOP06 model [CIE 2006], from which the CIE $20062^{\circ}$ and $10^{\circ} \mathrm{CMFs}$ were derived. Since this present study made use of a $3^{\circ}$ field (mirror size), the CIE $20062^{\circ}$ standard observer should be the more appropriate CMF set for computing chromaticity coordinates, which is supported by these plots. To investigate the size effect on color matching, we calculated respectively the $3^{\circ}$ (match field: mirror) and $5^{\circ}$ (mirror frame) CMFs using the CIEOP06 model.

The chromaticity of the color matches computed using the $3^{\circ}$ and $5^{\circ} \mathrm{CMFs}$ are shown in the Figure $4 \mathrm{e}$ and $4 \mathrm{f}$. The $3^{\circ} \mathrm{CMFs}$ provide the smallest metameric differences (closest clustering of mean matches and SE-ellipses) compared to the other CMF sets, even smaller than those computed with the CIEOP06 $2^{\circ}$ CMFs. This is consistent with the actual size of the match field used in the experiments. 
The matches from the reference matching (RM) session show very similar results, as can be observed from Figure 5. A comparison of Fig. 5 (RM matches) and Fig. 3 (MM matches) shows that the computed ellipses are smaller when matching to a spectrally broadband reference color. Increased variability (larger ellipses) for memory color matching can be explained by individual differences in what observers actual consider 'neutral grey', despite any differences in their visual perception. By providing a reference color to the observers, this additional variability is not present. However, the matched color itself could still vary depending on the primary set and CMFs set used. However, as can be seen from the clustering of the colored dots in Fig. 5, this effect was minimized by adopting the reference stimulus with a smooth broadband spectrum generated by the reflected light of a spectrally neutral grey material illuminated by a filtered halogen source.

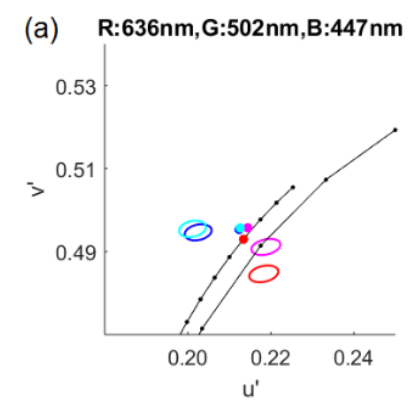

(d) R:636nm, G:502nm,B:404nm

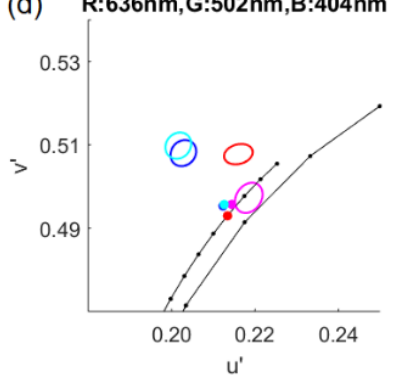

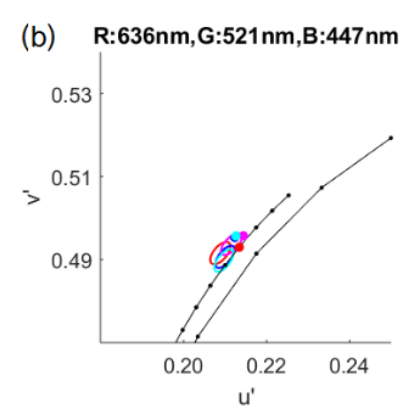

(e) R:636nm,G:521nm,B:404nm

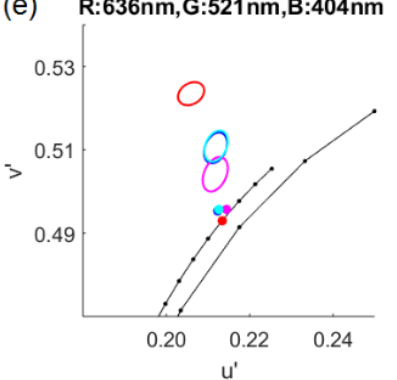

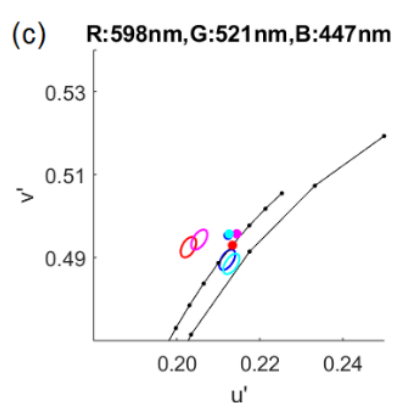

(f) R:598nm,G:521nm,B:404nm

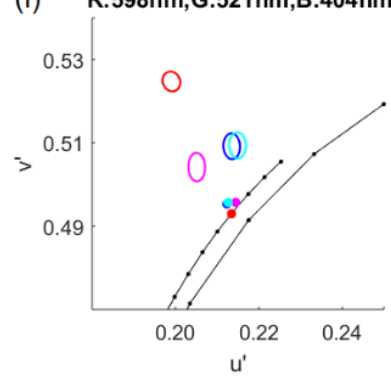

Figure 5-RM condition: Computed chromaticity coordinates of reference color (dots) and the SE ellipses of matches made with different primary sets (subplots a-f) and CMF sets (Red: CIE $19312^{\circ} \mathrm{CMFs}$, Blue: CIE $19641^{\circ} \mathrm{CMFs}$, Magenta: CIE $20062^{\circ} \mathrm{CMFs}$, Cyan: CIE $200610^{\circ}$ CMFs).

Figure 6 shows the distribution of match ellipses computed by different CMF sets and some similar results as in Fig. 4 can be observed. The two $10^{\circ} \mathrm{CMFs}$ (CIE $196410^{\circ} \mathrm{CMFs}$ and $\mathrm{CIE}$ $200610^{\circ} \mathrm{CMFs}$ ) again show very similar predicted matches (i.e. similar position, size, shape and orientation of the SE ellipses). The CIE $19312^{\circ}$ CMFs again results in the largest spread of matching ellipses across the 6 primary-sets. However, in the reference matching session, the CIE $20062^{\circ} \mathrm{CMFs}$ do not predict the color matches as well as CIEOP06 $5^{\circ} \mathrm{CMFs}$. As can be seen in Figs. 6 , the chromaticity of the matches computed with the CIEOP06 $3^{\circ}$ and $5^{\circ} \mathrm{CMFs}$ are more clustered together for the different primary sets, with the $3^{\circ} \mathrm{CMFs}$ having the best performance. This result agrees with the size of the match field $\left(3^{\circ}\right.$ mirror $)$. However, none of the tested CMFs can perfectly predict the matches across all the primary sets because the chromaticity differences between mean matches using certain different primary sets are larger than one JND. 

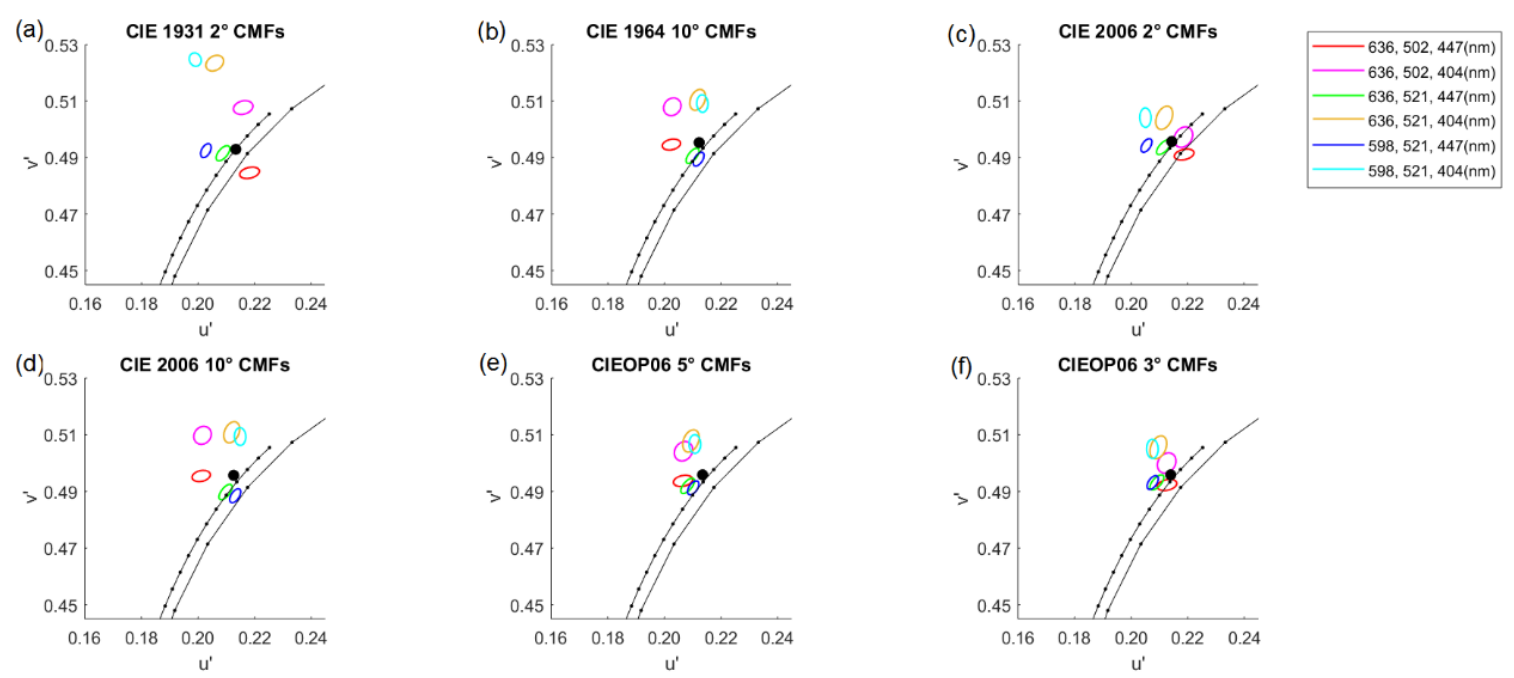

Figure 6 -RM condition: Chromaticity coordinates of reference matches (black dots) and the SE ellipses of matches made with different primary-set (subplots a-f), and correlated primarysets (6 colors indicate 6 different primary-sets).

In both of the experiment sessions, the results indicate that matches made by the primary set with peak wavelengths $636 \mathrm{~nm}, 521 \mathrm{~nm}$ and $447 \mathrm{~nm}$ are the most stable across changes in CMF set. These peak wavelengths are typical for LED RGB light sources [Csuti 2008] and are widely used in both research and industry [Asano 2013]. Among all the tested primaries, the wavelengths of this set are also closest to those of the monochromatic red $(650 \mathrm{~nm})$, green $(530 \mathrm{~nm})$ and blue $(460 \mathrm{~nm})$ primaries used by Wright [Wright 1929] to generate data in his $2^{\circ}$ color matching experiments, and to the peak wavelengths of the primaries $(645.2 \mathrm{~nm}, 526.3 \mathrm{~nm}$ and $444.4 \mathrm{~nm}$ ) adopted by Stiles \& Burch [Stiles 1959, 1955] in their $10^{\circ}$ color matching experiments. In addition, the Stiles \& Burch data with this primary set was also used by Stockman to develop a cone fundamental model [Stockman 1999, 2000]. As the later CIEOP06 [CIE 2006] model is mainly based on Stockman 2000's work, the CIE 2006 CMFs were also derived depending on these primaries. It is therefore not surprising that this particular primary set is stable across changes in CMFs set. Even though this primary set has quite stable matching performance, the metameric differences generated by using other primaries are obvious in the experiment results of the present study. Therefore, it is important to note that recent work on color matching [Sarkar 2010, 2011] - also adopting primaries with peak wavelengths close to the ones of this particular set - may have underestimated the discrepancies between visual and computed matches.

Finally, these results suggest that the field size has an obvious impact on color matching, as substantial metameric differences between visual and computed matches remain even when taking field size into account in the CIE 2006 CMFs. Therefore, in future work, it is important to further characterize the accuracy of existing CMFs and observer variability, and to consider a set of optimized color matching functions.

\section{Conclusion}

Experimental results show that the choice of a spectrally narrowband primary set has a clear impact on the chromaticity of the color match calculated using different CMF sets. Changing one of the primaries results in different shifts in the chromaticity calculated by the different CMFs. The same relative trends were observed for all experiment conditions. While there are substantial differences in the calculated match chromaticities, depending on the adopted spectrally narrowband matching primaries, the chromaticities calculated using the different CMF sets are very similar for the spectrally smooth and broadband reference grey. Calculated chromaticities for matches made by mixing the light of the $636 \mathrm{~nm}, 521 \mathrm{~nm}$ and $447 \mathrm{~nm}$ primaries, which are typical LED RGB-primaries [Csuti 2008], are found also found to be stable across changes in the CMF set used to calculate them. From all the test primary sets, this set has peak wavelengths closest to those adopted in the Wright [Wright 1929] and Stiles \& Burch [Stiles 1959, 1955] matching experiments which generated data that was used in the 
development of the CIE $19312^{\circ}$ and the CIE $196410^{\circ} \mathrm{CMFs}$, respectively. The data from Stiles \& Burch was also used in the development of Stockman's cone fundamental model [Stockman 1999, 2000] on which the CIEOP06 CMFs are based [CIE 2006]. It is therefore reasonable that this particular primary set can obtain the least discrepancy between visual and computed metamers. Furthermore, as recent work on color matching accuracy also adopted primary sets with wavelengths close to those of this stable set, the reported accuracy and variability of calculated matches could be severely underestimated.

In addition, the results show that all the existing CMF sets, even the latest CIE 2006 cone fundamental based CMFs were not strongly predictive for visual matches made with different narrow-band primaries. If basic colorimetry holds, for each individual participant, the matching color should be visually the same for different primary sets. For an average observer, the calculated tristimulus values should also match closely. The CIE $10^{\circ} 1964$ and CIEOP06 $10^{\circ}$ CMFs produced very similar match chromaticities, while those of the $2^{\circ} \mathrm{CIE} 1931$ and CIEOP06 differed much more. Substantial calculated chromaticity differences were found for the CIE 1931 $2^{\circ}$ standard observer CMFs, while the metameric differences generated by using the CIEOP06 $2^{\circ}$ CMFs were substantially smaller. The former was found to be the most different when a very short wavelength primary $(404 \mathrm{~nm})$ was used, confirming earlier reports on the problems of this CMF set. Considering that the match field size has an impact on color matching, CIEOP06 $3^{\circ}$ CMFs were calculated, and it was found to be the most predictive one in the results. However, substantial difference between visual and predicted matches was still observed by using CIEOP06 $3^{\circ}$ CMFs, which suggests the need for a further characterization of existing color matching models and CMFs and to develop a more accurate CMF set.

Future research will put basic colorimetry under test. A discovery of key problems and the derivation of a better colorimetric system would have important consequences not only for the prediction of color (mis)matches, but also for all colorimetric calculations based on CMFs, such as color difference equations, color appearance models, computer renderings and VR, etc. A better understanding of the extent of observer variability would also shed light and might (partially) explain the, often large, variability found in other colorimetric and psychological color variables.

\section{References}

Csuti, P. \& Schanda, J. A better description of metameric experience of LED clusters. Light Eng. 18, 44-50 (2010).

Csuti, P. \& Schanda, J. Colour matching experiments with RGB-LEDs. Color Res. Appl. 33, 108-112 (2008).

Flecy, L., Withouck, M. (cosup., Hanselaer, P. (cosup.., Smet, K. A. G. (sup. . \& Smet, K. A. G. RGB color matching with narrow- and broadbanded primaries". Master thesis, KU Leuven, Ghent, BE. ESAT Master in, (KU Leuven, 2015).

Ezquerro, J. M., Zoido, J. M., Perales, E., Mart'inez-Verd'u, F. \& Melgosa, M. Analysing observer metamerism in CIECAM02 using real observers. Conf. Colour Graph. Imaging, Vis. 2008.

Houser KW, Hu X. The UNL Trichromatic Colorimeter. Color Res Appl, 2005; 30:209-220.

Wright WD (1929). A re-determination of the trichromatic coefficients of the spectral colours. Trans. Opt. Soc. 30, 141.

Stiles, W. \& Burch, J. NPL colour -matching investigation: final report (1958). J. Mod. Opt. 6, 1-26 (1959).

Stiles WS, and Burch JM (1955). Interim Report to the Commission Internationale de l'Eclairage, Zurich, 1955, on the National Physical Laboratory's Investigation of Colour-matching (1955). Opt. Acta Int. J. Opt. 2, 168-181.

Stockman A, and Sharpe L (1999). Cone spectral sensitivities and color matching. In Color Vision: From Genes to Perception., K. Gegenfurtner, and L. Sharper, eds. (Cambridge: Cambridge University Press), pp. 53-57. 
Stockman A, and Sharpe LT (2000). The spectral sensitivities of the middle- and longwavelength- sensitive cones derived from measurements in observers of known genotype. Vision Res. 40, 1711- 1737.

CIE \& CIE. Fundamental Chromaticity Diagram with Physiological Axes - Part I. 170 1:2006, (CIE, 2006).

CIE15-2004 (2004). Colorimetry (Vienna, Austria: CIE). CIE15.2-1986.

Asano Y, Fairchild MD, and Blondé L (2013). Observer variability experiment using a fourprimary display and its relationship with physiological factors. In Proc. of CIC21, (Albuquerque, NM, USA: Society for Imaging Science and Technology), p. 6.

Sarkar, A., Autrusseau, F., Viénot, F., Le Callet, P. \& Blondé, L. From CIE 2006 physiological model to improved age-dependent and average colorimetric observers. J. Opt. Soc. Am. A 28, 2033-2048 (2011).

Sarkar, A. CIE report R1-54 "Variability in Color-matching Functions". (CIE, 2011).

Sarkar A, Blondé L, Le Callet P, Autrusseau F, Stauder J, and Morvan P (2009). Study of Observer Variability on Modern Display Colorimetry: Comparison of CIE 2006 Model and $10^{\circ}$ Standard Observer. In The 11th Congress of the International Colour Association (AIC), (Sydney, Australia: AIC), p.

Sarkar A, Blondé L, Callet P Le, Autrusseau F, Morvan P, and Stauder J (2010). Toward Reducing Observer Metamerism in Industrial Applications: Colorimetric Observer Categories and Observer Classification. In Proc. of CIC18, (San Antonio, Texas, USA, November 8-12, 2010.: Society for Imaging Science and Technology), pp. 307-313.

Rich DC, and Jalijali J (1995). Effects of observer metamerism in the determination of human color- matching functions. Color Res. Appl. 20, 29-35.

Smet KAG, Deconinck G, and Hanselaer P (2015). Chromaticity of unique white in illumination mode. Opt. Express 23, 12488-12495.

Smet K, Deconinck G, and Hanselaer P (2014). Chromaticity of unique white in object mode. Opt. Express 22, 25830-25841.ADRIAN, W. 1989. Visibility of Targets: Model for Calculation. Lighting Res. Technol., 21, 181-188. 